\section{$\underset{\substack{\text { hommes } \\ \text { \& migrations }}}{ }$}

\section{Hommes \& migrations}

Revue française de référence sur les dynamiques

migratoires

$1304 \mid 2013$

Frontières

\title{
Tanger et les harraga : les mutations d'un espace frontalier
}

\section{Sarah Przybyl et Youssef Ben Tayeb}

\section{(apenEdition \\ Journals}

\section{Édition électronique}

URL : http://journals.openedition.org/hommesmigrations/2636

DOI : 10.4000/hommesmigrations.2636

ISSN : 2262-3353

\section{Éditeur}

Musée national de l'histoire de l'immigration

\section{Édition imprimée}

Date de publication : 1 octobre 2013

Pagination : 41-48

ISBN : 978-2-919040-24-7

ISSN : $1142-852 X$

\section{Référence électronique}

Sarah Przybyl et Youssef Ben Tayeb, «Tanger et les harraga : les mutations d'un espace frontalier», Hommes \& migrations [En ligne], 1304 | 2013, mis en ligne le 01 janvier 2017, consulté le 02 mai 2019. URL : http://journals.openedition.org/hommesmigrations/2636 ; DOI : 10.4000/ hommesmigrations.2636 


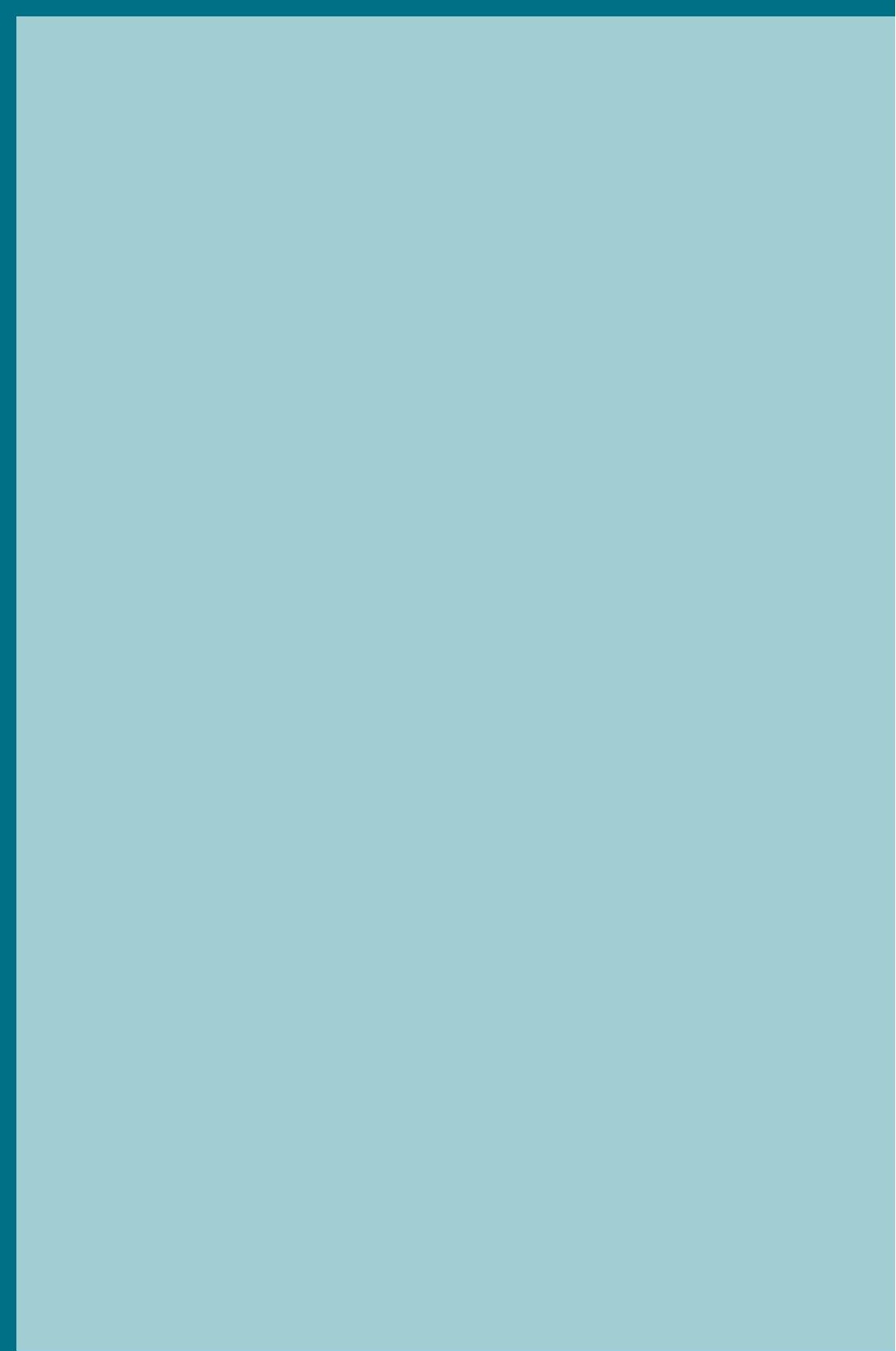

Série Territoire, Gilles Delmas, 2002

(c) Musée de l'histoire de l'immigration, Courtesy de l'artiste 


\title{
TANGER ET LES HARRAGA LES MUTATIONS D'UN ESPACE FRONTALIER
}

par SARAH PRZYBYL, doctorante en géographie au laboratoire Migrinter, université de Poitiers, et YOUSSEF BEN TAYEB, étudiant en master II professionnel Migrations internationales, laboratoire Migrinter, université de Poitiers.

\author{
Pour de nombreux jeunes migrants tentés par une traversée \\ clandestine, les ports de Tanger-ville et Tanger-Med au Maroc \\ constituent à la fois un refuge et un tremplin vers l'Europe. \\ Or les mutations récentes des infrastructures portuaires \\ modifient les projets et le mode de vie des harraga, à l'affût \\ des opportunités de passage. À Tanger, I'espace à la fois \\ clos et ouvert des ports joue le rôle d'une carte où se dessinent \\ les projets migratoires d'une jeunesse en mal d'avenir.
}

\section{Tanger-Med, un espace hautement sécurisé mais non moins attractif}

Les harragas ${ }^{1}$, visage inédit de l'émigration clandestine marocaine depuis une vingtaine d'années, se composent majoritairement de mineurs originaires du sud du pays (province de Béni Mellal), de Casablanca (quartier périphérique de Sidi Moumen) et de Tanger. Chadia Arab définit le hrague ${ }^{2}$ comme "le voyage vers l'eldorado européen, dans la soute d'un bateau, dans un conteneur ou une barque de passeurs après un passage clandestin au Maroc ${ }^{3 "}$. Protégés par la Convention internationale des droits de l'enfant, ces mineurs sont considérés comme délinquants, l'émigration par des voies clandestines étant illégale ${ }^{4}$ dans la législation marocaine. Dans ce contexte, et voulant profiter de sa position 
stratégique sur le détroit de Gibraltar, le Royaume a réalisé un nouveau pari : se placer parmi les leaders mondiaux des échanges maritimes, avec la mise en activité du nouveau complexe portuaire de Tanger-Méditerranée (et plus récemment de son annexe Tanger-Med II). Simultanément, le port historique de Tanger-ville tend à devenir un port de plaisance débarrassé des poids lourds.

L'ouverture de cette nouvelle porte en direction de l'eldorado européen nous a conduits à étudier la façon dont les changements en termes d'infrastructures portuaires s'ac-

Si, pour le Royaume,

Tanger-Med est un pari prometteur, pour les candidats au hrague ce port marque

l'ouverture d'un lieu de passage vers l'Europe. compagnent de mutations dans les dynamiques de départ des harragas et d'une redéfinition des espaces portuaires. Au mois de mars 2013 (hors période estivale), c'est en binôme que nous sommes allés enquêter auprès des jeunes candidats à l'émigration clandestine en misant sur des échanges informels lors des temps d'attente et d'observation, souvent synonymes d'immobilité dans les espaces portuaires tangérois.

Le géant portuaire Tanger-Med se présente depuis sa mise en activité en 2007 comme une nouvelle "porte d'entrée vers le Maroc 5 ". Bâtissant sa réputation de port nouvelle génération à travers la promotion de l'efficacité et de la rapidité de ses échanges, Tanger-Med s'est doté d'un dispositif de sécurité portuaire considérable. L'omniprésence de caméras (classiques, infrarouges et optiques), de radars, de détecteurs de battements de cœur, de clôtures ou encore de contrôle automatiques des accès piétons indique la préoccupation des autorités portuaires à l'égard des questions migratoires, et plus particulièrement sous leurs formes clandestines.

$\mathrm{Si}$, pour le Royaume, Tanger-Med est un pari prometteur, pour les candidats au hrague ce port marque l'ouverture d'un lieu de passage vers l'Europe. C'est dans cette frontière paradoxale, largement ouverte, créatrice de nouvelles opportunités de départs mais dans le même temps fermement clôturée, réduisant de fait les possibilités de passage, que s'inscrivent les tentatives de traversées clandestines des harraga. Malgré l'apparente inaccessibilité du lieu et l'impossibilité de se jouer des systèmes de contrôle, des jeunes ont dompté une partie de la mécanique du géant portuaire pour parvenir à embarquer vers le continent européen. Les premiers mineurs ayant réussi le passage, "ceux qui l'ont fait", font office de pionniers venant nourrir un horizon des possibles pour des nouveaux arrivants confrontés à l'allure infranchissable de cette frontière sous surveillance. TangerMed est donc un lieu investi de figures de harraga emblématiques, de traversées presque légendaires et d'anecdotes sur les imprévus du voyage qui se retrouvent dans les discours des jeunes en attente de pouvoir partir à leur tour.

Malgré son verrouillage, Tanger-Med n'en reste pas moins attractif au regard du nombre de camions qui y transitent. Au-delà des éléments objectivement repérables qui, à première vue, font de ce port une enceinte impénétrable, ce sont bien les manières dont les jeunes investissent ce lieu qui en font un espace ouvert au passage.

Les histoires de hrig qui se transmettent, ou qui sont en train de s'écrire, constituent un faisceau d'indices permettant de saisir la capacité des harraga à dépasser ces points infranchissables de la frontière.

\section{Vivre pour partir : profils de harraga rencontrés à Tanger-Med}

Lors de l'enquête de terrain, nous sommes restés avec une dizaine de mineurs originaires de Sidi Moumen $^{6}$. Nous les avons rencontrés alors qu'ils mendiaient auprès des chauffeurs de poids lourds sortant de la zone portuaire. Les membres de ce petit groupe ont grandi ensemble. Âgés de 14 à 18 ans, ils sont d'anciens voisins, des cousins ou les membres d'une fratrie. Si les raisons qui les 
ont poussés à quitter leur quartier d'origine sont diverses (difficultés familiales, violence, pauvreté, absence de perspectives d'avenir...), c'est bien ensemble qu'ils ont décidé de vivre les différentes étapes du voyage avant de tenter de partir vers l'Europe depuis Tanger-Med. Effectuée en groupe, une première migration interne de Sidi Moumen vers Tanger-ville met en action le projet migratoire de ces jeunes.

En constatant le rétrécissement des opportunités dans le port historique de Tanger-ville, les membres de ce groupe ont décidé de s'installer dans l'antre de Tanger-Med avec l'espoir de quitter le Maroc, ce qui induit une organisation sociale et spatiale dictée par des règles mises en place par les candidats à l'émigration qui, sortis de leur environnement habituel recréent une microsociété dont ils sont les protagonistes et qui tend à rendre réalisable le hrig, objectif ultime de leur présence. Vivre dans cet espace frontalier tangérois les contraint à une vie rythmée par la mendicité, les membres du groupe se relayant pour mendier, sous la surveillance du plus âgé.

Tanger-Med se partage. Le groupe rencontré n'étant pas le seul à vouloir partir, on observe une occupation stratégique des lieux vacants de la colline où les mineurs s'abritent.

Deux organisations spatiales hiérarchisées sont repérables : d'une part, entre les groupes de harraga présents qui se partagent l'espace (au regard de l'ancienneté des jeunes casablancais sur le port de Tanger-Med, ces derniers se situent au plus près du port et bénéficient donc de la position la plus stratégique en cas de départ); d'autre part, au sein même du groupe de harraga enquêtés, ce qui donne une forme pyramidale basée sur l'âge des mineurs, les plus âgés se trouvant au sommet de la colline et détenant l'argent récolté. En descendant, l'âge diminue et les installations se font de plus en plus sommaires.

Au-delà de l'expression spatiale d'un rapport de force entre les membres des différents groupes, ces installations répondent à une logique de sécurité dans la mesure où, d'après les mineurs, les policiers n'arrêtent que très peu les plus jeunes, ce sont les plus âgés d'entre eux qui subiraient un traitement plus sévère.

Dans un autre ordre d'idées, en parcourant les cavités de la colline, on remarque que ce lieu, vivant au rythme des essais de hrague et des activités liées à la survie, présente des éléments d'un environnement connu des jeunes. Des similitudes apparaissent dans la manière de progresser dans le territoire du groupe de Casablancais et celui du groupe du bidonville de Sidi Moumen : les draps et les couvertures tendus qu'il faut balayer de la main pour progresser, la sinuosité des passages à emprunter, mais aussi la pénombre des petites grottes où s'abritent les mineurs. Tout se passe comme si, dans cet environnement soumis aux aléas des tentatives de départ, des interventions des forces de police et des difficultés de la subsistance quotidienne, les jeunes donnaient à cette frontière un air familier et sécurisant leur permettant de penser et de réaliser le départ. Tanger-Med en tant que nouvelle porte de la frontière tangéroise s'inscrit comme un point relais après une première migration interne depuis les régions d'origine des mineurs. Bien plus qu'une simple étape, c'est un lieu ressource, un premier refuge dans lequel les jeunes recréent un cadre sécurisant répondant aux

Tout se passe comme si, dans cet environnement soumis aux aléas des tentatives de départ, des interventions des forces de police et des difficultés de la subsistance quotidienne, les jeunes donnaient à cette frontière un air familier et sécurisant leur permettant de penser et de réaliser le départ.

règles qu'ils instaurent et où semblent avoir été évacuées les problématiques quotidiennes qui ont pu les pousser à quitter leur région. Ce lieu représente à la fois un tremplin vers le "rêve européen" et un refuge où les formes de solidarité sont génératrices de liens entre les harraga qui élaborent ensemble des stratégies d'accès aux côtes européennes, deuxième refuge à atteindre. 


\section{De Tanger-ville à "Tanger-vide" ?}

Face à l'émergence de Tanger-Med, Tanger-ville, vidé en grande partie des camions et des bus touristiques à destination des côtes espagnoles, perd sa réputation de port emblématique des départs clandestins pour renforcer celle de "perle du détroit ${ }^{8}$. Comme le montrent les photos, le port de Tanger-ville semble vide depuis la réalisation du projet de reconversion. Si la baisse du nombre de camions induit Les mutations du port

de Tanger-ville ont fait ainsi

reculer la frontière dans

les méandres de la médina

tangéroise, participant dans

le même temps à la rupture

d'une frontière linéaire

aux limites claires et définies. une réduction des opportunités de passage, marque-telle pour autant la diminution du nombre de harraga et de tentatives d'émigration clandestine depuis ce port? Plusieurs conclusions ont pu être tirées au sujet de cet espace portuaire. Tout d'abord, c'est par la configuration du port qu'une transition s'est opérée en termes de passage pour les jeunes candidats à l'émigration. Si autrefois le franchissement du portail bien gardé menant au port était pour les harraga un premier pas vers l'Europe, à l'heure actuelle la destruction de l'enceinte a eu pour corollaire d'en faire reculer les limites et de renforcer les moyens humains de contrôle d'accès au port ${ }^{9}$. Or, dans le même temps, la vigilance des autorités semble aléatoire. Au regard de l'absence de possibilités de passage, il semblerait que l'intrusion de mineurs dans le port soit tolérée certains jours.

Le constat dressé à Tanger-ville permet dans un second temps de conclure que, même s'il semble que le port se soit vidé (en partie) des harraga, ce n'est pas parce que ces derniers sont moins nombreux qu'avant le projet de reconversion, mais parce qu'ils sont moins visibles. Cette non-visibilité dans l'enceinte portuaire est liée à la délocalisation des points de départ des mineurs. La dimi-

nution du nombre de poids lourds restreint les possibilités de passages clandestins, de sorte que chaque bus ou camion en partance pour l'Europe depuis Tanger-ville représente une opportunité à ne pas rater. Les jeunes candidats à l'émigration sont en mouvement dans différents quartiers de la ville, où sont implantés les hôtels accueillant des touristes majoritairement européens, dans l'attente de pouvoir se cacher dans tous les types de véhicules. Les techniques de hrague ne connaissent pas d'évolution majeure, néanmoins la reconversion du port et la disparition d'une entrée unique vers l'Europe ont eu pour conséquence une multiplication, un éclatement et une délocalisation des points de passage. Les mutations du port de Tanger-ville ont fait ainsi reculer la frontière dans les méandres de la médina tangéroise, participant dans le même temps à la rupture d'une frontière linéaire aux limites claires et définies. L'impression d'un "Tanger-vide" en est le corollaire direct. Elle est renforcée par la circulation incessante des mineurs dans la médina comme moyen d'optimiser les chances de passage. Mentionnons que des jeunes tentent toujours le passage clandestin depuis le port de Tanger-ville en recourant à des techniques de plus en plus risquées.

\section{Vers une diversification des profils et une redéfinition de l'espace portuaire}

L'éclatement de la frontière dans différents lieux de Tanger, lié à la mise en marche du projet de reconversion, ne donne qu'une vision partielle des dynamiques de l'émigration clandestine. Les temps du port dictent une temporalité des opportunités de passage, la présence plus ou moins accrue des harraga dans l'enceinte portuaire en est un indicateur. La période estivale marque un accroissement de l'activité dans le port de Tanger-ville : bus, voitures et bateaux à quai sont alors plus nombreux, ce qui multiplie les interstices dans lesquels les jeunes 
peuvent se glisser pour "risquer ${ }^{10 "}$. Le nombre de mineurs sur le port augmente alors considérablement ${ }^{11}$. Tout se passe comme si "Tanger-vide", décrit précédemment comme un lieu peu propice au départ, redevenait, l'espace de quelques semaines, un tremplin assuré vers le continent européen.

Lors des observations quotidiennes, l'échelle hebdomadaire a permis de lire le jeu d'ouverture et de fermeture du port de Tanger-ville d'une part, et de fournir l'explication de la fréquentation très aléatoire des mineurs d'autre part. Durant la semaine, on note pendant deux jours une hausse de la fréquentation du port par les mineurs candidats à l'émigration, hausse directement liée à l'augmentation des bus en attente d'embarquement et de débarquement lors du chassécroisé de la clientèle touristique résidant dans les complexes hôteliers tangérois.

À l'inverse, les forces de l'ordre multiplient les arrestations des jeunes qui se trouvent à l'intérieur et aux alentours de l'enceinte portuaire le jour de la visite hebdomadaire (supposée) du préfet, afin de faire place nette au représentant du roi. Dans le même ordre d'idées, en raison d'une baisse de l'activité de l'ensemble du personnel travaillant dans le port et, de fait, du transit, le jour saint ${ }^{12}$, le vendredi, s'inscrit nettement comme un temps de ralentissement des échanges. De ce fait, les mineurs ne sont que très peu présents dans le port et font de ce jour un moment de répit.

Avant l'émergence de Tanger-Med et le projet de reconversion de Tanger-ville, le port faisait l'objet d'une occupation de jour comme de nuit par les jeunes qui, en restant plus longtemps, augmentaient leurs chances de passage : enfants des rues et harraga vivaient alors ensemble dans le port. À l'heure actuelle et d'après nos observations, en dehors de la période estivale, seuls les enfants des rues habitent les abords du port.

\section{Tentatives de migration et rite de passage}

Pour comprendre l'émigration clandestine des mineurs marocains, il est nécessaire de distinguer enfants des rues et harraga afin d'éviter la corrélation hâtive entre errance et émigration. Même si l'errance constitue encore un mode de vie pour certains d'entre eux, la majeure partie des mineurs candidats à l'émigration clandestine rencontrés durant la période d'enquête sont très éloignés de la

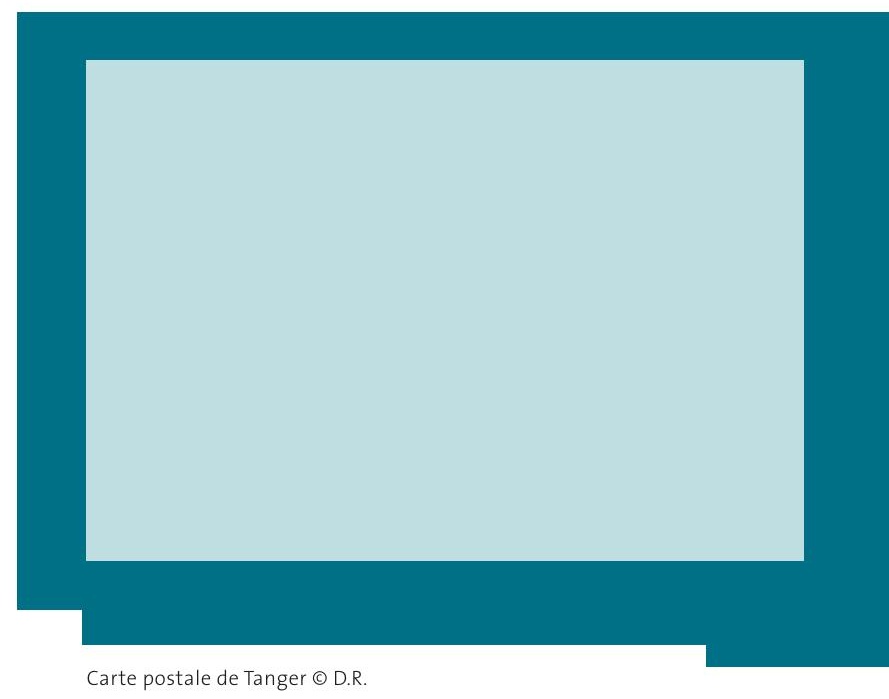

figure des enfants des rues. Prenons pour exemple Nabil, 12 ans, qui, portant encore son sac d'écolier sur l'épaule, nous avoue avoir fait l'école buissonnière et être venu voir s'il pouvait tenter le hrig avant de rentrer chez lui le soir venu. Beaucoup de témoignages de mineurs vivant dans leur famille, scolarisés et originaires de Tanger, sont similaires aux propos tenus par Nabil ; ce qui se joue dans le franchissement de cette frontière va au-delà d'un changement d'État. Pour des jeunes comme Nabil, 
passer la limite de l'enceinte portuaire et "risquer" signent la ritualisation du changement d'état ${ }^{13}$, celui de l'adolescent à l'adulte. La transgression, le départ (ponctuel) du foyer familial, l'inscription dans un groupe de pairs, l'apprentissage des techniques de passage ou encore la reproduction d'attitudes des plus âgés sont à l'heure actuelle autant d'éléments qui montrent que, plus qu'une migration, se jouent dans cette frontière les scènes transitoires du passage à l'âge adulte. Le témoignage de Nabil et de bien d'autres sont une invitation à reconsidérer la catégorie de harraga à la lumière de ces différents visages que nous avons pu croiser sur le port de Tanger-ville, mais aussi à repenser la frontière comme le terrain d'une transition fondamentale qui se joue pour certains mineurs venant s'essayer au hrig. Au regard

La mobilité entre de la baisse des opportuniles deux enceintes portuaires tés de passage, une mise à se présente comme distance de cette frontière une ressource que mobilisent les jeunes à un moment de leur parcours, dans l'objectif d'optimiser leurs chances de passage. polymorphe s'opère. Le port tend à ne plus être un lieu de vie (ou devrionsnous dire de survie) pour devenir celui de la tentative, fréquenté de manière opportune par les harragas. La frontière portuaire tangéroise est donc devenue progressivement un des lieux constitutifs de l'espace de vie ${ }^{14}$ des différents mineurs candidats à l'émigration clandestine rencontrés. Tout se passe comme si le savoir-migrer s'accompagnait d'un savoir-grandir, où l'inscription spatiale était constitutive d'une transition plus globale des jeunes présents.

\section{La mobilité comme ressource}

Face à la réduction des opportunités et à l'accroissement des échanges entre harraga, il convient de s'interroger sur l'existence de circulations entre le port de Tanger-ville et Tanger-Med comme une réponse au resserrement des possibilités de hrig observées par les mineurs. Parmi les histoires entendues, l'une illustre particulièrement ce qui se joue dans cet espace frontalier, celle d'Omar et Tariq, deux frères âgés de 15 et 16 ans originaires des bidonvilles tangérois. Après maintes réussites de passages, ils ont été systématiquement renvoyés vers le Maroc: "Ça fait plusieurs années que je vis dans les rues de Tanger, j’ai connu une époque où on pouvait partir facilement. Depuis, il n'y a plus de camions, les possibilités de hrig sont limitées et, aujourd'hui, le peu de bus qui passent au port de Tanger sont pris par tous les harraga et ça fait souvent des disputes. En plus, ils surveillent tout le temps les bus, au port ou aux hôtels, et ça, ça nous rend la tâche plus difficile. C'est pour ça que nous, on se cache sous les camions qui vont à Tanger-Med, parce que là-bas il y a tellement de camions que nous avons plus de chances."

La mobilité entre les deux enceintes portuaires se présente comme une ressource que mobilisent les jeunes à un moment de leur parcours, dans l'objectif d'optimiser leurs chances de passage. La circulation illustre ici une prise de conscience réactivant le projet migratoire face à une situation ne pouvant offrir les opportunités attendues par les jeunes en situation d'attente sur le port de Tangerville. La mobilité peut être considérée comme une simulation précédant la tentative. En effet, pour certains mineurs, les techniques de passage ne sont que théoriques et résultent d'une transmission par d'autres jeunes. Aussi, la mobilité entre ces deux points névralgiques de la frontière sous un camion peut-elle s'apparenter à un apprentissage des éléments essentiels à toute traversée clandestine par camion.

Si la mobilité physique entre les deux ports est avérée, ces allers-retours marquent l'échange d'informations entre les jeunes candidats à l'émigration qui, au retour de leur passage à Tanger-Med 
(des mineurs partent uniquement en repérage et reviennent le soir venu à Tangerville), viennent donner des informations, avérées ou non, sur le géant portuaire. Il existe des stratégies d'optimisation des chances de passage, dont une consiste à divulguer ou à cacher des informations cruciales sur les possibilités d'émigration clandestine dans les ports de la frontière tangéroise.

\section{Ceux qui ne parviennent pas à partir}

Après avoir distingué enfants des rues et candidats à l'émigration, il convient de s'arrêter sur les jeunes qui connaissent aujourd'hui une errance liée à l'échec d'une tentative d'émigration clandestine. Certaines informations que nous avons obtenues dans le cadre d'échanges avec des harraga indiquent que l'abandon du projet migratoire peut constituer l'événement déclencheur d'une vie d'errance de durée variable.

Ahmed, 17 ans : "Il y a un ami de mon quartier qui a réussi à passer la semaine dernière et la nouvelle s'est répandue. Depuis, j'ai rejoint des amis déjà là-bas et ça fait deux jours que je veille et que je ne suis pas allé travailler, mais je suis fatigué et je vais rentrer chez moi."

Pour une partie des jeunes rencontrés, la vie dans la rue comme moyen d'optimiser les chances de hrig ne s'étend pas sur une longue période, mais c'est un instant en suspens au cours duquel observation et attente se mêlent dans l'espoir de saisir le moment optimal pour traverser clandestinement le détroit. Cette étape provisoire que beaucoup de jeunes expérimentent avant d'atteindre les côtes européennes s'accompagne d'une frustration. Pour des adolescents comme Ahmed, cette lassitude de l'attente se traduit par un retour au quartier d'origine ; pour d'autres, la rue devient un mode de vie fait de débrouille et d'errance.

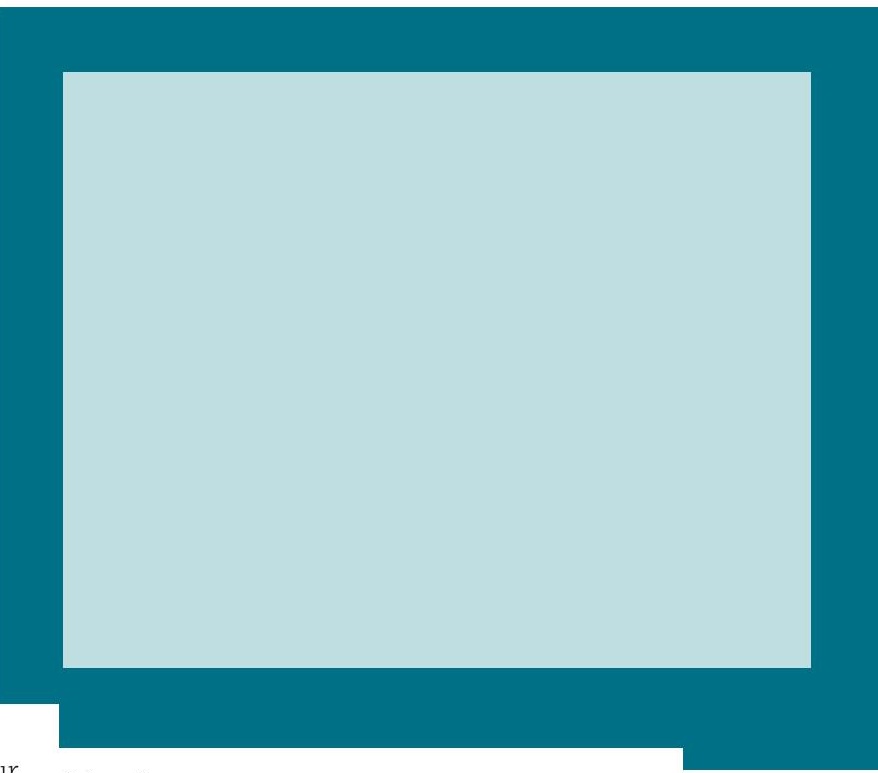

(c) SARAH PRZYBYL

Tel est le cas de Nordine, 17 ans, originaire de Meknès, qui a quitté sa famille à l'âge de 10 ans. Après avoir affronté le décès de son père et des violences de la part de sa belle-mère, il se dirige vers Tanger-ville dans l'espoir d'effectuer la traversée vers le continent européen. Constatant le peu d'opportunités, Nordine bascule dans une vie d'errance où mendicité et usage de drogues remplacent tout désir d'émigration : "Aujourd'hui, je mendie pour avoir suffisamment La rencontre avec le monde de la rue peut marquer l'abandon de tout projet d'argent pour pouvoir m'acheter du diluant pour décoller et moublier."

Nicolas Mai explique que, pour les mineurs, le besoin d'avenir, qu'il soit pensé au Maroc ou en Europe. Les rêves de certains adolescents semblent s'être évaporés dans les vapeurs des diluants. de dépasser un sentiment de frustration entraîne le recours à des produits qui procurent une satisfaction immédiate. Les plans à long terme sont donc évincés au profit d'une jouissance ponctuelle ${ }^{15}$. Pour les mineurs vivants dans la rue à la suite de l'abandon de leur projet migratoire, il subsiste une urgence à oublier les trauma- 
tismes passés, parfois à l'origine du désir de partir. Les pratiques illicites repérées sur le terrain sont autant de réponses à ces besoins et viennent se substituer au projet migratoire. Pour une partie de ces adolescents, le recours à la drogue s'explique par un besoin d'oublier la violence quotidienne qu'implique la vie dans la rue ; l'usage de produits permet d'effectuer un voyage, celui de l'esprit, comme l'ersatz d'une traversée impossible à réaliser depuis le port de Tanger-ville. "Décoller", comme dit Nordine, est devenu le quotidien de beaucoup de jeunes, mais parmi ceux ayant abandonné l'idée de partir, tous ne sont pas devenus chamkara ${ }^{16}$.

La rencontre avec le monde de la rue peut marquer l'abandon de tout projet d'avenir, qu'il soit pensé au Maroc ou en Europe. Les rêves de certains adolescents semblent s'être évaporés dans les vapeurs des diluants.

À d'autres, cette période d'errance donne une raison supplémentaire de vouloir quitter le Maroc et tenter le passage clandestin vers l'Europe jusquà la réussite, ou encore, comme en témoigne Ahmed de simplement retourner dans sa famille. Il est fondamental de comprendre l'hétérogénéité des processus à l'œuvre afin de saisir les incidences de la rencontre entre certains mineurs marocains et la frontière tangéroise.

\section{Conclusion}

À Tanger, la frontière connaît actuellement de profondes mutations en termes d'équipements mais aussi de gestion du transit des marchandises et des personnes. Les harraga s'inscrivent dans cette nouvelle donne frontalière. L'observation des infrastructures portuaires a démontré un jeu d'ouverture et de fermeture de la frontière. À travers nos observations Tanger-Med et Tanger-ville font partie intégrante de l'espace de vie des mineurs candidats à l'émigration clandestine et d'autres jeunes dans une plus large mesure. Refuge, porte vers un ailleurs prometteur, lieu d'abandon de soi, terrain d'une transition adolescente, espace de confrontation aux pairs, les ports étudiés sont des scènes sur lesquelles se jouent différents scénarios aux issues incertaines. Les profils des harraga en sont une illustration de par leur diversité, les aléas du quotidien venant redistribuer constamment les cartes du destin de ces jeunes. Analyser l'espace frontalier tangérois conduit à considérer les autres frontières, celles des inégalités de la société marocaine, moins visibles mais synonymes d'absence de perspectives d'avenir pour une partie de la jeunesse marocaine. 